\title{
A soluble tau fragment generated by caspase-2 is associated with dementia in Lewy body disease
}

Benjamin R. Smith ${ }^{1,2}$, Kathryn M. Nelson ${ }^{3}$, Lisa J. Kemper ${ }^{1,2}$, Kailee Leinonen-Wright ${ }^{1,2}$, Ashley Petersen ${ }^{4}$, C. Dirk Keene $e^{5}$ and Karen H. Ashe $e^{1,2,6^{*}}$

\begin{abstract}
Lewy body diseases are neurodegenerative disorders characterized by Lewy bodies in the brain. Lewy body dementia (LBD) refers to two forms of Lewy body disease: Parkinson's disease with dementia (PDD) and dementia with Lewy bodies (DLB). Tau is a cytoskeletal protein found in neurofibrillary tangles, but not Lewy bodies. The gene encoding tau, MAPT, is a well-established genetic risk factor for LBD; odds ratios of the H1:H2 MAPT haplotypes have been reported in the range of 2 to 4 . Despite this genetic association, the mechanism by which tau contributes to dementia is unclear. Recently, a soluble form of tau, $\Delta$ tau314, which is generated when caspase2 (Casp2) cleaves tau at Asp314, was reported to be associated with impaired cognition in mice modeling frontotemporal dementia, and with mild cognitive impairment and Alzheimer's disease (AD) in humans. To investigate whether $\Delta$ tau314 is associated with dementia in Lewy body disease, we compared $\Delta$ tau314 levels in aqueous extracts from the superior temporal gyrus of pathologically confirmed LBD $(n=21)$ and non-dementia Parkinson's disease (PD) $(n=12)$. We excluded subjects with AD or microvascular pathology, which could mask potential associations of $\Delta$ tau314 with LBD.

Using a $\Delta$ tau314-specific ELISA, we found $\sim 2$-fold higher levels of $\Delta$ tau314 in LBD relative to PD $(p=0.009)$. Additionally, we found $\sim 40 \%$ lower levels of soluble total tau and the neuronal marker $\beta$-III-tubulin in LBD. These results suggest that in $L B D$, there is substantial neuron loss or axonal degeneration in the neocortex but disproportionately high levels of $\Delta$ tau314 in the surviving neurons.

Our results indicate an association between $\Delta$ tau314 and dementia in Lewy body disease. Cleavage of tau by Casp2 promotes the mislocalization of tau to dendritic spines leading to a reduction in postsynaptic AMPA receptors and excitatory neurotransmission, which suggests a mechanism of the synaptic dysfunction underlying cognitive impairment in LBD. These findings support the potential of Casp2 as a novel drug target for treating LBD.
\end{abstract}

Keywords: Lewy body, Dementia, Parkinson's, Caspase-2, Tau

\section{Introduction}

Dementia is a frequent feature of Lewy body disease, a class of neurodegenerative conditions characterized by the presence of Lewy bodies in the brain. Lewy bodies are cytoplasmic inclusions containing $\alpha$-synuclein proteins. Lewy body dementia (LBD) refers to demented patients who have Lewy body pathology. The two forms

\footnotetext{
* Correspondence: hsiao005@umn.edu

${ }^{1} \mathrm{~N}$. Bud Grossman Center for Memory Research and Care, University of Minnesota, 2101 6th Street SE, Minneapolis 55455, USA

${ }^{2}$ Department of Neurology, University of Minnesota, Minneapolis, USA Full list of author information is available at the end of the article
}

of LBD - Parkinson's disease with dementia (PDD) and dementia with Lewy bodies (DLB)-differ in clinical presentation. Dementia appears before or within 1 year of motor signs in DLB, while motor signs appear 1 year before dementia in PDD. There is a strong but imperfect association between neocortical Lewy bodies and dementia. Neocortical Lewy bodies were found in $49.5 \%$ of patients with PDD and $15.2 \%$ of patients with Parkinson's disease without dementia (PD) [10].

Tau is a microtubule-associated protein encoded by the MAPT gene on Chr17q21.31. There are two major alleles of Chr17q21.31, termed $\mathrm{H} 1$ and $\mathrm{H} 2$. The H2 haplotype

(c) The Author(s). 2019 Open Access This article is distributed under the terms of the Creative Commons Attribution 4.0 International License (http://creativecommons.org/licenses/by/4.0/), which permits unrestricted use, distribution, and 
arose through the inversion of a $\sim 900 \mathrm{~Kb}$ segment of Chr17q21.31, which spans several genes, including MAPT. Many GWAS studies of LBD show a protective effect of the $\mathrm{H} 2$ allele. The $\mathrm{H} 1: \mathrm{H} 2$ odds ratio (OR) for PDD versus PD was reported to be 3.7 [16]. In PD, homozygosity for $\mathrm{H} 1$ was associated with accelerated progression to dementia [7]. The H1:H2 OR for DLB versus controls was reported to range from 2.3-3.3 [12]. However, in a larger study [8] MAPT showed only nominal association.

Tau is an axonal protein whose normal function includes promoting the polymerization of tubulin to form microtubules in axons. Under disease conditions, tau redistributes to the soma and dendrites, and insoluble tau accumulates in cytoplasmic neurofibrillary tangles. Using tau positron tomography to image neurofibrillary pathology in humans, increased cortical uptake was found in LBD relative to normal control subjects [6].

Soluble forms of tau also play pathogenic roles. A soluble tau fragment, $\Delta$ tau314, forms when caspase-2 (Casp2) cleaves tau at Asp314 [23]. Rendering tau resistant to Casp2 cleavage preserves memory function, and lowering Casp2 restores memory function in mice expressing mutant human tau linked to frontotemporal dementia [23]. Cleavage and phosphorylation of tau lead to tau mislocalization to dendritic spines, reduced AMPA receptor levels in the postsynaptic membrane, and abnormal excitatory neurotransmission [9, 23]. Cells lacking Casp2 or expressing Casp2-resistant tau do not show tau mislocalization or impaired synaptic transmission [23].

Casp2 is a member of the family of cysteine aspartic proteases, of which there are 12 in humans. Casp2 has both apoptotic and non-apoptotic roles, the latter including the modulation of autophagy and cell cycle arrest. Cleavage of tau to generate $\Delta$ tau 314 is another non-apoptotic effect of Casp2.

The levels of $\Delta$ tau314 were $\sim 3$-fold higher in brain extracts from patients with mild cognitive impairment (MCI) and Alzheimer's disease (AD) relative to cognitively intact subjects [23], suggesting that $\Delta$ tau314 may be a biomarker for impaired cognition. The relationship between $\Delta$ tau314 and cognition has never been examined in Lewy body disease. Here we tested the hypothesis that $\Delta$ tau314 is associated with dementia in Lewy body disease, independent of $\mathrm{AD}$ or microvascular pathology.

\section{Materials and methods}

\section{Aim, design and setting of the study}

The aim of the study was to compare levels of $\Delta$ tau 314 in patients with Lewy body disease with (i.e., LBD) and without dementia (i.e., PD), in the absence of $\mathrm{AD}$ or microvascular pathology. We obtained frozen brain specimens from the superior temporal gyrus of donated brains from research participants in the Pacific Udall
Center, the Alzheimer's Disease Research Center, and the Adult Changes in Thought Study from the University of Washington Neuropathology Core. At autopsy, every brain underwent comprehensive neuropathological evaluation per the latest diagnostic guidelines for the assessment of Alzheimer's disease neuropathologic change, Lewy body disease, vascular brain injury, and other disorders. Lewy body disease specimens were selected using the following criteria: presence of Lewy bodies in the brainstem, limbic area, or neocortex; absence of a "moderate" or "frequent" CERAD neuritic amyloid plaque score; and few or no microvascular lesions. A clinical diagnosis of dementia was assigned based on psychometric testing and formal review at consensus conferences specific to each study. A total of 21 LBD and 12 PD specimens met these criteria.

\section{Demographics and clinical characteristics of the subject population}

There was no significant difference in age $(\mathrm{PD}=82.8$; $\mathrm{LBD}=77.3)$, sex $(\mathrm{PD}=58 \%$ male; $\mathrm{LBD}=81 \%$ male $)$, $\mathrm{PMI}$ $(\mathrm{PD}=5.5 ; \mathrm{LBD}=6.3)$, brain weight $(\mathrm{PD}=1231 \mathrm{~g} ; \mathrm{LBD}=$ $1339 \mathrm{~g})$, or months since last evaluation $(\mathrm{PD}=31.3$; $\mathrm{LBD}=45.8$ ) between the groups (Table 1 ).

\section{Pathological characteristics of the subject population}

All subjects had Lewy body disease. The distribution of Lewy bodies in the brain in the PD and LBD groups differed significantly (Table 2). All subjects had sparse or no neuritic amyloid plaques and Braak Stages $\leq 4$. There were no significant differences in Braak Stages or CERAD scores between the LBD and PD groups (Table 2).

\section{Biochemical extraction}

Superior temporal gyrus was collected at the University of Washington fresh during rapid autopsies, flash frozen in liquid nitrogen, and stored at $-80 \mathrm{C}$ until the samples were shipped on dry ice to the University of Minnesota. Neverthawed superior temporal gyrus samples were extracted in Tris-buffered saline (TBS) containing $50 \mathrm{mM}$ Tris- $\mathrm{HCl}$ (pH 7.4), $150 \mathrm{mM} \mathrm{NaCl}$, protease inhibitor cocktail, $1 \mathrm{mM}$ phenylmethylsulfonyl fluoride, $1 \mathrm{mM}$ phenanthroline monohydrate and phosphatase inhibitors. Homogenates were centrifuged at $16,000 \mathrm{~g}$ for $60 \mathrm{~min}$ to pellet nuclei and large debris, and the supernatant was collected.

\section{APOE genotyping}

Human brain tissue was homogenized and DNA was extracted using Qiagen DNeasy Blood and Tissue Kit (Cat 69506). DNA was quantified using Thermo Scientific NanoDrop spectrophotometer. APOE status was determined using APOE c.388 T $>\mathrm{C}$ and APOE c.526C $>\mathrm{T}$ Novallele Genotyping Assays (Canon BioMedical Inc. Cat40394 and Cat 40395) run on a Roche Lightcycler 480 system. 
Table 1 Demographic and clinical characteristics of subject population

\begin{tabular}{|c|c|c|c|c|}
\hline & PD & PDD & Total & $p$ value \\
\hline \multicolumn{5}{|l|}{ Age (years) } \\
\hline Group size & 12 & 21 & 33 & \\
\hline Mean \pm SD & $82.8 \pm 10.5$ & $77.3 \pm 13.1$ & $79.3 \pm 12.4$ & \\
\hline Range & $57-98$ & $42-96$ & $42-98$ & $0.19^{a}$ \\
\hline \multicolumn{5}{|l|}{ Sex } \\
\hline Group size & 12 & 21 & 33 & \\
\hline Male & 7 & 17 & 24 & \\
\hline Female & 5 & 4 & 9 & $0.23^{b}$ \\
\hline \multicolumn{5}{|l|}{ PMI (h) } \\
\hline Group size* & 11 & 21 & 32 & \\
\hline Mean \pm SD & $5.5 \pm 2.2$ & $6.3 \pm 4.0$ & $6.0 \pm 3.5$ & \\
\hline Range & $1.9-11$ & $2-16.3$ & $1.9-16.3$ & $0.45^{\mathrm{a}}$ \\
\hline \multicolumn{5}{|l|}{ Brain weight (g) } \\
\hline Group size* & 11 & 17 & 28 & \\
\hline Mean \pm SD & $1231 \pm 131$ & $1339 \pm 236$ & $1296 \pm 205$ & \\
\hline Range & $1010-1450$ & $1070-2017$ & 1010-2017 & $0.14^{\mathrm{a}}$ \\
\hline \multicolumn{5}{|c|}{ Last evaluation (months) } \\
\hline Group size & 8 & 14 & 22 & \\
\hline Mean \pm SD & $31.3 \pm 35.4$ & $45.8 \pm 29.1$ & $40.5 \pm 31.5$ & \\
\hline Range & $15.3-118.5$ & $10.2-98.6$ & $10.2-118.5$ & $0.34^{\mathrm{a}}$ \\
\hline
\end{tabular}

aunpaired t-test with Welch's correction

${ }^{\mathrm{b}}$ Fisher's exact test

*Data for one or more patient was unavailable

\section{$\Delta$ tau314 and Total tau measurements by enzyme-linked} Sandwich immunoassay

Samples were analyzed using Quanterix ${ }^{\mathrm{TM}}$ ultra-sensitive, single molecule array (Simoa) technology. 4F3 monoclonal antibodies were custom-generated by Precision Antibodies $^{\mathrm{TM}}$. $\Delta$ tau314 measurements were performed using 4F3-coated magnetic beads for protein capture and a combination of biotinylated BT2/HT7 antibodies for detection. Total tau (T-tau) was measured using Tau 2.0 kits $\left(\right.$ Quanterix $\left.^{\mathrm{TM}}\right)$. All measurements were performed in triplicate on the same day in the same run using the same reagents, and experimenters were blind to clinical status.

\section{$\beta$-III-tubulin and GAPDH measurements by Western blotting}

TBS extracts were normalized to protein levels (BCA assay, Thermo Fisher), diluted in reducing sample buffer, size-fractionated by SDS-PAGE on $10 \%$ Tris- $\mathrm{HCl}$ gels (Bio-Rad), and transferred onto $0.2 \mu \mathrm{m}$ nitrocellulose membranes. Immunoblots were probed with $\beta$-III-tubulin (Sigma Aldrich) and GAPDH (Cell Signaling) antibodies and visualized using chemiluminescence reagents (Pierce) followed by exposure onto autoradiography film (Gene Mate). Band densities were measured using Fiji
Table 2 Pathological characteristics of subject population

\begin{tabular}{lllll}
\hline & PD & PDD & Total & $p$ value \\
\hline Lewy bodies & & & & \\
Group size & 12 & 21 & 33 & \\
Neocortical & $3(25 \%)$ & $18(86 \%)$ & $21(64 \%)$ & \\
Limbic & $3(35 \%)$ & $0(0 \%)$ & $3(9 \%)$ & \\
Brainstem & $6(50 \%)$ & $3(14 \%)$ & $9(27 \%)$ & $0.001^{\text {a }}$ \\
Group size* & 11 & 20 & 31 & \\
Braak 0 & $0(0 \%)$ & $2(10 \%)$ & $2(6 \%)$ & \\
Braak 1 & $3(27 \%)$ & $5(25 \%)$ & $8(26 \%)$ & \\
Braak 2 & $3(27 \%)$ & $2(10 \%)$ & $5(16 \%)$ & \\
Braak 3 & $3(27 \%)$ & $6(30 \%)$ & $9(29 \%)$ & \\
Braak 4 & $2(18 \%)$ & $5(25 \%)$ & $7(23 \%)$ & \\
Braak 5 & $0(0 \%)$ & $0(0 \%)$ & $0(0 \%)$ & \\
Braak 6 & $0(0 \%)$ & $0(0 \%)$ & $0(0 \%)$ & $0.86^{\mathrm{a}}$ \\
CERAD & & & & \\
Group size* & 12 & 20 & 32 & \\
None & $1(8 \%)$ & $8(40 \%)$ & $9(28 \%)$ & \\
Sparse & $11(92 \%)$ & $12(60 \%)$ & $23(72 \%)$ & $0.10^{\mathrm{b}}$ \\
\hline aChi-squared test & & & & \\
bisher's exact test & & &
\end{tabular}

software (NIH). TBS extracts for Casp2 detection were run on $10.5-14 \%$ gradient gels (Bio-Rad) and visualized using a Casp2 specific antibody (Abcam).

\section{Statistical analysis}

Groups were compared using the Mann-Whitney test or Student's two-tailed t-tests with Welch's correction. In addition, a multiple linear regression model was fitted with the logarithm of measured values ( $\Delta$ tau314, total tau (T-tau), or $\Delta$ tau314: T- tau) and age, sex, and PMI. $P$-values for the comparisons are presented when using a two-sample t-test on the logged measure, which is equivalent to the linear regression with no adjustment variables. Demographics were analyzed using unpaired ttest with Welch's correction or Fisher's exact test. Pathological characteristics were analyzed using $X$-squared or Fisher's exact tests. AUC's were compared using DeLong's test for correlated ROC curves.

\section{Results}

Soluble $\Delta$ tau314 is higher in Lewy body dementia (LBD) than non-dementia Parkinson's disease (PD)

We tested the hypothesis that $\Delta$ tau314 is a biomarker for dementia in Lewy body disease. We measured $\Delta$ tau314 in aqueous extracts from the superior temporal gyrus of 12 PD and 21 LBD patients, and found $~ 2$-fold higher levels of $\Delta$ tau314 in LBD $(5.035 ; 2.696-10.95 \mathrm{ng} / \mathrm{g})$ than PD (2.59; 1.16-4.163 ng/g) (Fig. 1a). (Note: In this paper, all 


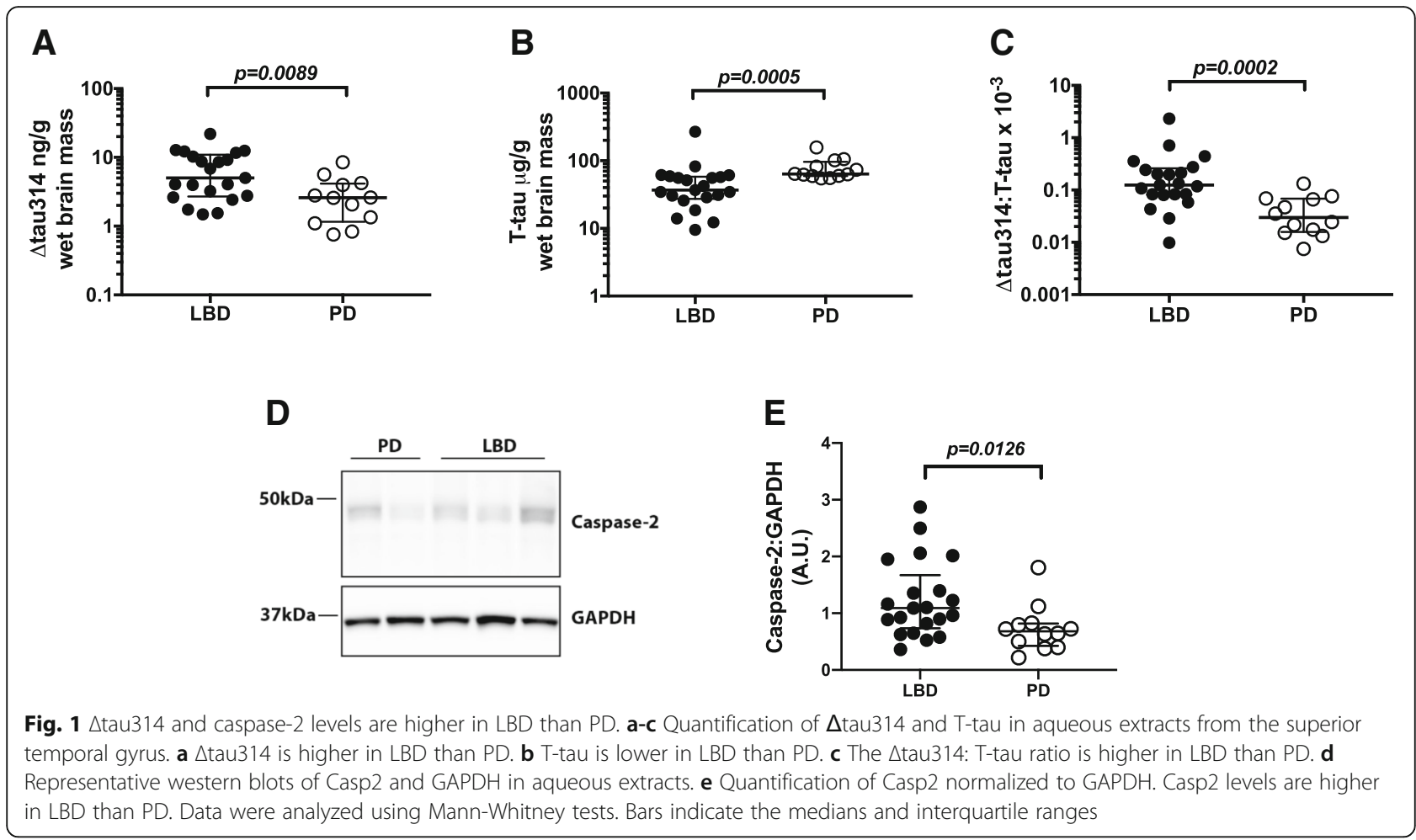

tau measurements are presented as the amount of the protein of interest relative to wet brain mass, and the numerical values represent median; first - third interquartile intervals.) Applying either a Mann-Whitney test, two-tailed t-test with Welch's correction, or multiple regression model adjusted for age, sex, and PMI, we found that the levels of $\Delta$ tau314 were significantly higher in LBD than PD (Table 3).

\section{Caspase-2 is higher in LBD than PD}

Casp2 is produced as a zymogen containing a caspase activation and recruitment domain (CARD) situated in the amino-terminus of the protease domain. Like other caspases, proteolytic removal of the CARD activates Casp2. However, oligomerization of the intact zymogen is also sufficient to activate Casp2 [3]. At present, there are no reliable techniques for measuring Casp2 activity directly in human brain tissue. Therefore, we measured
Casp2 levels as a proxy for Casp2 activity. To assess Casp2 levels we used western blotting to measure levels of the $48 \mathrm{kDa}$ zymogen, and found $\sim 59 \%$ higher levels of Casp2 in LBD than PD (Fig. 1d, e). Elevated levels of Casp2 are also observed in AD $[11,18]$, suggesting LBD and $\mathrm{AD}$ share a common pathway leading to increased levels of Casp2.

\section{Soluble total tau is lower in LBD than PD}

To determine whether higher levels of $\Delta$ tau314 in LBD were due to higher levels of soluble tau, we measured total tau (T-tau) by ELISA in the same aqueous extracts. Surprisingly, we found $\sim 40 \%$ lower levels of T-tau in LBD $(36.88 ; 27.32-58.1 \mu \mathrm{g} / \mathrm{g})$ than PD $(63.58 ; 59.54-$ $96.48 \mu \mathrm{g} / \mathrm{g}$ ) (Fig. 1b). The reduced levels of T-tau resulted in $\sim 4$-fold higher levels of $\Delta$ tau314 normalized to T-tau in LBD (0.1248; 0.08135-0.2593) versus PD (0.02991; 0.01575-0.0684) (Fig. 1c). All comparisons

Table 3 Statistics for Fig. 1. Data were analyzed using two-tailed unpaired non-parametric Mann-Whitney test, two-tailed t-test, and multiple-regression model adjusted for age, sex, and post-mortem interval

\begin{tabular}{llll}
\hline & Mann-Whitney test & Two-sample t-test & $\begin{array}{c}\text { Multiple linear regression } \\
\text { (adjusted for age, sex, PMl) }\end{array}$ \\
\hline$\Delta$ tau314 & $p=0.0089$ & $p=0.0075$ & $p=0.0229$ \\
Total tau & $p=0.00049$ & $p=0.0012$ & $p=0.0090$ \\
$\Delta$ tau314/Total tau & $p=0.00024$ & $p=0.00026$ & $p=0.0020$ \\
\hline
\end{tabular}


remained significant after adjustment for age, sex, and PMI via multiple linear regression (Table 3).

\section{Neuronal marker $\beta$-III-tubulin is lower in LBD than PD}

To determine whether lower levels of T-tau in LBD was due to neuron loss or axonal degeneration, we analyzed $\beta$-III-tubulin in the same aqueous extracts by SDS-PAGE and western blotting (Fig. 2a). GAPDH levels did not differ between LBD and PD groups, and thus served as a loading control (Fig. 2b). We found a $50 \%$ decrease in $\beta$-III- tubulin levels in LBD relative to PD, suggesting significant numbers of neurons or axons are lost in the superior temporal gyrus of LBD relative to PD patients (Fig. 2c). There was no difference in the T-tau: $\beta$-III-tubulin ratio between LBD and PD (Fig. 2d), indicating that the decrease in T-tau is attributable largely to neuron or axon loss. The $\Delta$ tau314:( $\beta$-III-tubulin:GAPDH) ratio was $\sim 7$-fold higher in LBD than PD (Fig. 2e), reflecting disproportionately high levels of $\Delta$ tau 314 in the surviving neurons.

\section{APOE $\varepsilon 4$ is associated with higher levels of $\Delta$ tau314}

Although APOE status is not associated with PD diagnosis [5], APOE $\varepsilon 4$ is a genetic risk factor for LBD [8]. The $\varepsilon 4$ allele of APOE is a strong risk factor for DLB, conferring an odds ratio of 2.4-6.1 $[8,21]$. The APOE $\varepsilon 4$ allele frequency is higher in PDD and DLB groups than in normal subjects or non-demented PD patients [14, 21]. Consistent with prior reports, in this study an APOE $\varepsilon 4$ allele was present in 7 out of 21 LBD patients, but no PD patients.

To detect a possible association between $\Delta$ tau 314 and APOE, we compared $\Delta$ tau 314 levels in APOE $\varepsilon 4$ carriers and non-carriers. We first examined the subjects with LBD, and found no significant differences in the levels of $\Delta$ tau314 (Fig. 3a). However, we found an $\sim 2$-fold higher ratio of $\Delta$ tau314: T-tau in APOE $\varepsilon 4$ carriers $(0.2751$; $0.1178-0.7082 ; n=7)$ compared to non-carriers $(0.1162$; $0.07089-0.205 ; n=14$ ) (Fig. 3b).

When we analyzed LBD and PD subjects together, we found a trend toward higher levels of $\Delta$ tau314 in APOE $\varepsilon 4$ carriers $(8.667 ; 4.064-11.63 \mathrm{ng} / \mathrm{g} ; n=7)$ relative to non-carriers (3.376; $1.988-7.265 \mathrm{ng} / \mathrm{g} ; n=26)$ (Fig. $3 \mathrm{c}$ ), and an $\sim 4$-fold higher $\Delta$ tau314: T-tau ratio in APOE $\varepsilon 4$ carriers $(0.2751 ; 0.1178-0.7082)$ compared to non-carriers (0.0726; 0.02375-0.1328) (Fig. 3d). T-tau levels were lower in APOE $\varepsilon 4$ carriers (Fig. 4a, c).

To determine whether $\Delta$ tau314 associates with dementia independently of APOE $\varepsilon 4$, we compared $\Delta$ tau314 in demented (LDB) and cognitively intact (PD) APOE $\varepsilon 4$ non-carriers. We found $\sim 2$-fold higher levels in LDB $(4.55 ; 2.57-12.27 \mathrm{ng} / \mathrm{g} ; n=14)$ than PD $(2.59$; $1.16-4.16 \mathrm{ng} / \mathrm{g} ; n=12$ ) (Fig. 3e, f), indicating that factors besides APOE $\varepsilon 4$ status influence the association between $\Delta$ tau314 and dementia in Lewy body disease. In APOE $\varepsilon 4$ non-carriers, T-tau was $33 \%$ lower in LBD $(49.7 \mu \mathrm{g} / \mathrm{g}$ wet brain weight) than PD $(74.1 \mu \mathrm{g} / \mathrm{g}$ wet brain weight) patients. (Fig. 4e).
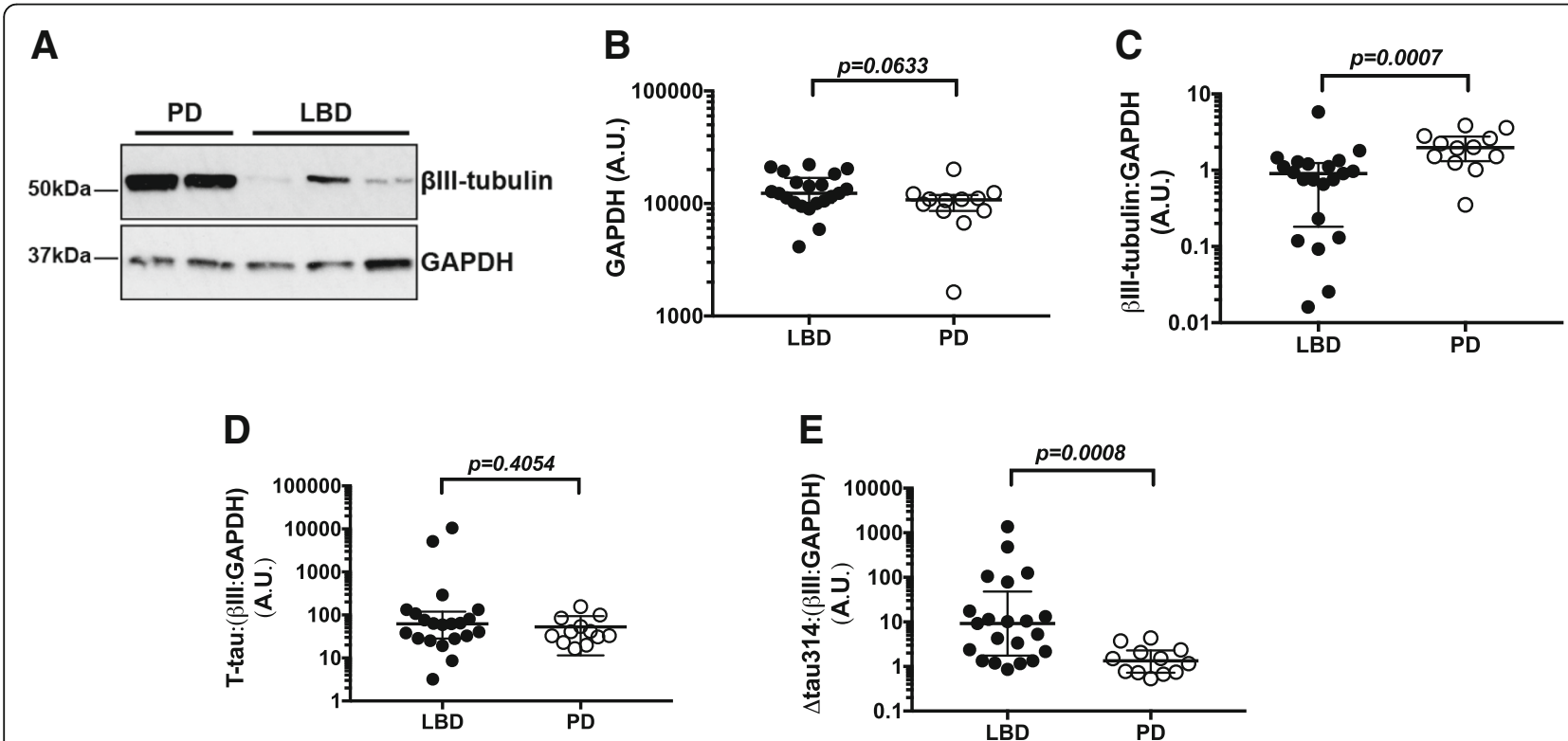

Fig. 2 Levels of $\beta$-III-tubulin are lower in LBD than PD. a Representative western blots of $\beta$-III-tubulin and GAPDH in aqueous extracts. b No significant difference in GAPDH levels between LBD and PD. GAPDH was used as a loading control. c Quantification of $\beta$-III-tubulin normalized to GAPDH. Normalized $\beta$-III-tubulin levels are lower in LBD than PD. $\mathbf{d}$ T-tau normalized to normalized tubulin ( $\beta$-III: GAPDH). No difference in normalized T-tau levels. e $\triangle$ tau314 normalized to normalized $\beta$-III-tubulin are higher in LBD than PD. Data were analyzed using Mann-Whitney tests. Bars indicate medians and interquartile ranges 

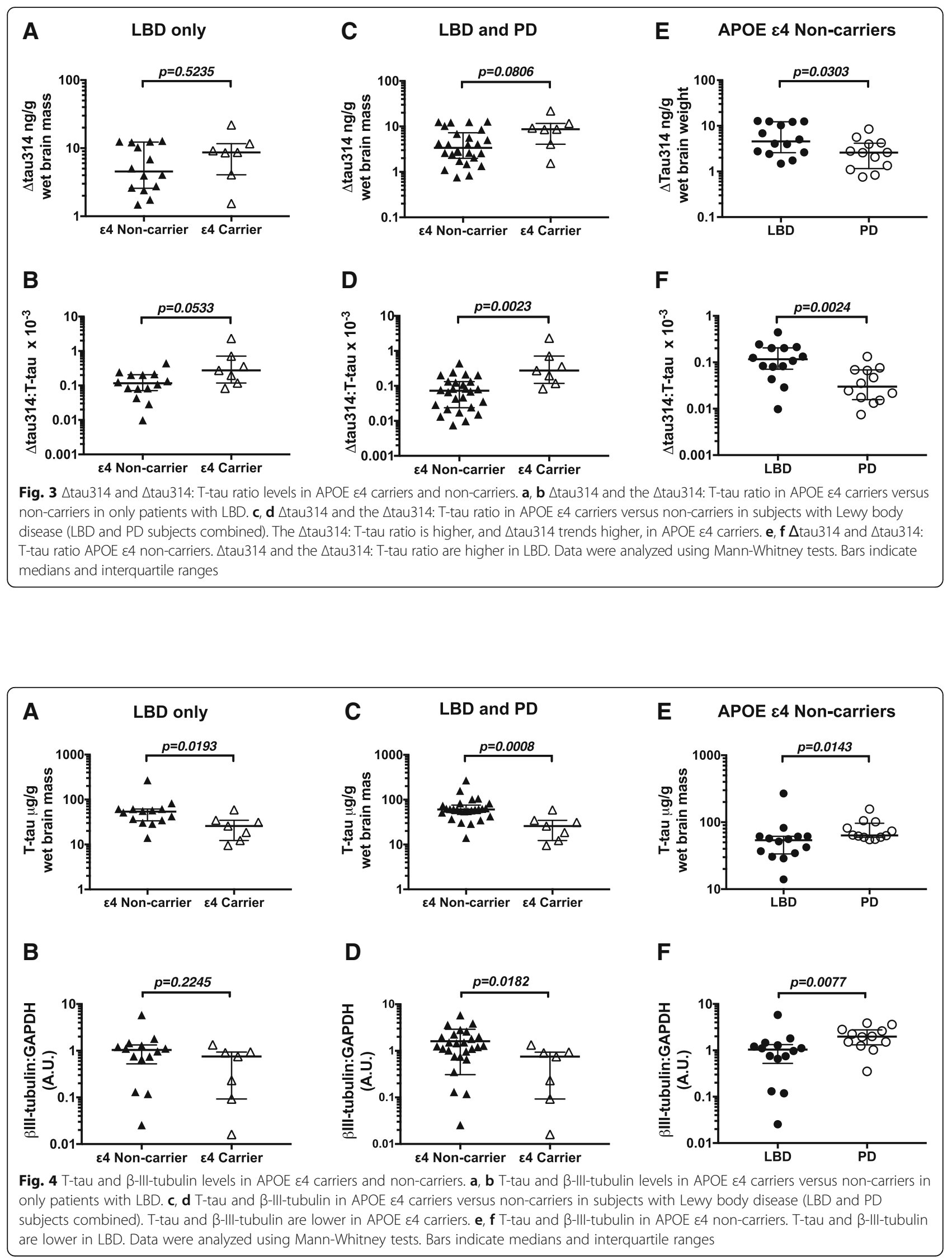
To establish whether neocortical neuronal or axonal loss is associated with APOE status, we compared $\beta$-III-tubulin levels in APOE $\varepsilon 4$ carriers and non-carriers. There was no difference in $\beta$-III-tubulin (normalized to GAPDH) levels in LBD (Fig. 4b). However, we found $\beta$-III-tubulin levels (normalized to GAPDH) were $62 \%$ lower in APOE $\varepsilon 4$ carriers than non-carriers when subjects were grouped together (Fig. 4d). Among APOE $\varepsilon 4$ non-carriers, $\beta$-III-tubulin levels in LBD were $40 \%$ lower than in PD (Fig. 4f), indicating that while APOE $\varepsilon 4$ status may contribute to neuronal or axonal loss it is not the sole factor.

\section{Receiver operating characteristics of $\Delta$ tau314, neurofibrillary tangles and Lewy bodies}

To assess the predicative value for dementia of $\Delta$ tau314, we generated receiver operating characteristic (ROC) curves. The AUC was 0.7738 for $\Delta$ tau314 (Fig. 5a) and 0.869 for the $\Delta$ tau314: T-tau ratio (Fig. 5b). The AUCs for $\Delta$ tau314: T-tau ratio $(\mathrm{AUC}=0.869), \Delta$ tau314 $(\mathrm{AUC}=.7738)$ and Lewy body staging $(\mathrm{AUC}=0.7857)$ did not differ significantly (Fig. 5c, Table 4). Braak staging, a measure of the distribution of neurofibrillary pathology, was not predictive
(Fig. 5d, Table 4), consistent with the exclusion of subjects with $\mathrm{AD}$ pathology from the study cohort.

\section{Discussion}

The soluble tau proteolytic fragment $\Delta$ tau314 is formed specifically when Casp2 cleaves tau at Asp314, and is associated with the disruption of postsynaptic excitatory neurotransmission. Here, we measured $\Delta$ tau314 in aqueous extracts of the superior temporal gyrus in subjects with Lewy body disease, and compared its levels with regard to the presence of dementia (i.e., Lewy body dementia, LBD) or the absence of dementia (i.e., Parkinson's disease, PD). We found higher levels of $\Delta$ tau314 in LBD than PD, and corresponding decreases in soluble T-tau and $\beta$-III-tubulin, a molecular proxy for neuron number or axon density. In addition, we found that Casp2 levels are elevated in LBD compared to PD; Casp2 levels in AD are higher than in controls, suggesting that $\mathrm{AD}$ and LBD share a common mechanism leading to increased Casp2 levels $[11,18]$. These results suggest that in LBD there is pronounced cortical neuron loss or axonal degeneration with disproportionately high levels of $\Delta$ tau314 in the remaining neurons. ROC analyses showed that $\Delta$ tau314
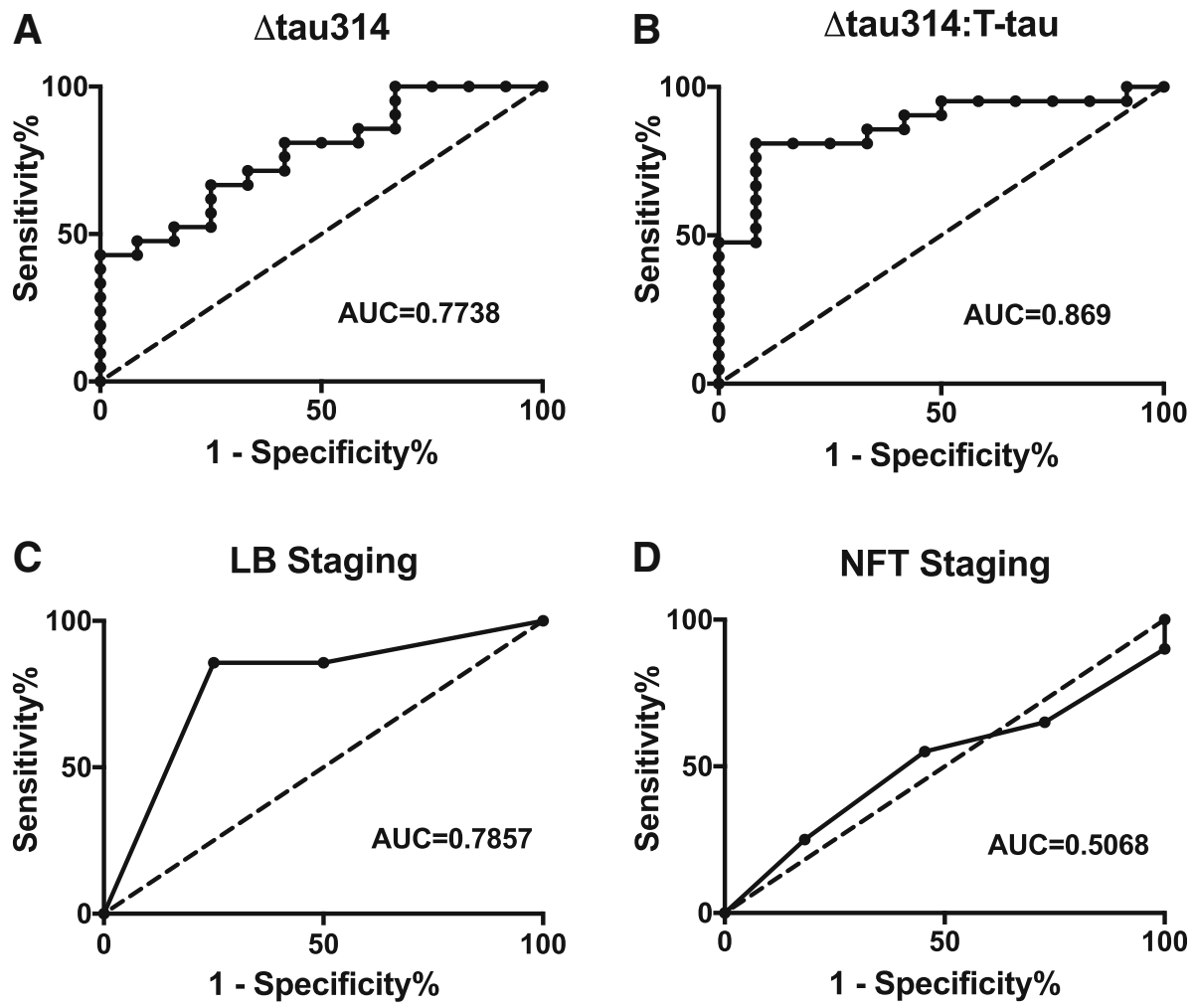

Fig. $5 \mathrm{ROC}$ analyses of $\triangle$ tau314, $\triangle$ tau314: T-tau, Lewy body staging, and neurofibrillary tangle staging in LBD versus PD. ROC curve analysis of a $\triangle$ tau314, b $\Delta$ tau314: T-tau, c Lewy body (LB) staging, and $\mathbf{d}$ neurofibrillary tangle (NFT) staging (using Braak criteria). The predictive value for dementia of $\triangle$ tau314, $\triangle$ tau314: T-tau and Lewy body staging are comparable, and superior to NFT staging. Dotted line is the line of identity $(A \cup C=0.5)$ 
Table 4 Statistics for Fig. 3. Data were analyzed using Delong's. Method for comparison of receiver operating characteristic curves

\begin{tabular}{ll}
\hline ROC comparison & $P$-value \\
\hline$\Delta$ tau314 vs. $\Delta$ tau314:total tau & 0.12 \\
$\Delta$ tau314 vs. LB stages & 0.92 \\
$\Delta$ tau314 vs. NFT stages & 0.11 \\
$\Delta$ tau314:total tau vs. LB stages & 0.45 \\
$\Delta$ tau314:total tau vs. NFT stages & 0.007 \\
\hline
\end{tabular}

performed as a predictor of dementia as effectively as Lewy body staging, the current gold standard.

We previously found increased levels of $\Delta$ tau314 in the inferior temporal gyrus of subjects with MCI and AD compared to normal controls [23]. Recently, we found increased levels of $\Delta$ tau314 in the prefrontal cortex and the caudate nucleus of patients with Huntington's disease (HD) compared to control subjects (Liu et al., Acta Neuropathologica Communications, this issue). Taken together with the findings presented here, we speculate that in $\mathrm{AD}, \mathrm{HD}$, and Lewy body disease, cognition becomes impaired when $\Delta$ tau314 accumulates in the neocortex. Our observation that $\Delta$ tau314 and the $\Delta$ tau314: T-tau ratio are equivalent to Lewy body staging for predicting dementia provides a strong rationale for attempting to measure $\Delta$ tau314 in blood or CSF, and for determining its potential utility as a molecular biomarker of synaptic dysfunction in living subjects.

The association of LBD with elevated levels of $\Delta$ tau314 is a novel finding. The importance of this result is that it lends support to the potential use of Casp2 inhibitors to ameliorate dementia in LBD. Several factors make Casp2 an attractive drug target. Not only does lowering Casp2 protect against several forms of neuronal insults, the absence of Casp2 has relatively few deleterious effects, at least in mice. The main adverse effects in Casp2 knockout mice are age-related bone and body fat loss and impaired hair growth [22]. Intraocular QPI-1007, an experimental siRNA against Casp2 used to treat nonarteritic anterior ischemic optic neuropathy, has thus far shown no serious harmful effects in clinical trials [1]. These features make targeting Casp2 a viable option for treating CNS diseases.

Casp2 has been implicated in mouse models of four neurodegenerative diseases -the $\mathrm{rTg} 4510$ model of frontotemporal dementia (FTD) [23], which expresses mutant human tau, the J20 mouse model of AD [15], which expresses mutant human APP, the YAC128 mouse model of HD [2], which expresses mutant human huntingtin, and the MPTP mouse model of Parkinson's disease (PD) [19]. Lowering Casp2 using an antisense oligomer decreased $\Delta$ tau314 production and improved memory function in $\operatorname{rTg} 4510$ mice, and prevented the mislocalization of tau to dendritic spines and reductions in excitatory postsynaptic neurotransmission [23]. Genetically ablating Casp2 in J20 mice reduced abnormal dendritic spine morphology and cognitive dysfunction [15], and conferred resistance to A $\beta$-mediated neurotoxicity in cultured neurons [20]. Knocking-out Casp2 in YAC128 mice ameliorated cognitive deficits, although neurodegeneration was not affected [2]. Casp2 knockout mice resisted neurotoxicity induced by the nigrostriatal neurotoxin MPTP [19].

APOE status affected the levels of neocortical $\Delta$ tau314, T-tau and $\beta$-III-tubulin. APOE $\varepsilon 4$ carriers showed more profound neuron loss or axonal degeneration, but disproportionately higher levels of $\Delta$ tau 314 in the remaining neurons. APOE $\varepsilon 4$ has been shown to increase tau-mediated neurodegeneration in P301S mice, and in several human tauopathies [17]. Interestingly, APOE $\varepsilon 4$ can induce mitochondrial dysfunction and oxidative stress [4], and mitochondrial oxidative stress can, in turn, activate Casp2 [13]. Thus, it is possible that APOE $\varepsilon 4$ may act in concert with mitochondria to increase Casp2 activity, leading to $\Delta$ tau314 production and neurodegeneration. APOE $\varepsilon 4$ is not exclusively responsible for neurodegeneration, however. Even in APOE $\varepsilon 4$ non-carriers, LBD patients had higher levels of $\Delta$ tau314 and lower levels of $\beta$-III-tubulin than PD patients, indicating that factors other than APOE also influence neuronal viability and $\Delta$ tau314 production.

The main limitations of our study are the group sizes and the sampling of one brain region only. Group sizes ranged from 12 to 21, and only the superior temporal gyrus was examined. A study of different brain regions in a larger cohort is needed to confirm our results. It would also be interesting to test the idea that $\Delta$ tau314 may correlate with the severity of motor signs in brainstem regions that are classically associated with Lewy body disease, such as the substantia nigra and striatum.

\section{Conclusion}

In this study, we report the novel finding that the Casp2generated tau fragment $\Delta$ tau314 is associated with dementia in Lewy body diseases. Our results suggest a mechanism for the synaptic dysfunction underlying dementia in these disorders, and support the development of Casp2 inhibitors to treat Lewy body dementia.

\section{Abbreviations}

AD: Alzheimer's disease; Casp2: Caspase-2; DLB: Dementia with Lewy bodies; FTD: Frontotemporal dementia; HD: Huntington's disease; LBD: Lewy body with dementia; $\mathrm{MCl}$ : Mild cognitive impairment; OR: Odds ratio; PD: Parkinson's disease; PDD: Parkinson's disease with dementia; T-tau: Total tau 


\section{Acknowledgements}

We acknowledge Peng Liu for insightful discussions, and Elizabeth Steuer for editorial assistance. We thank Allison Bellerm research coordinator for the University of Washington Neuropathology Core, which is supported by funding from NIH grants P50AG005136 (UW ADRC), U01AG006781 (Adult Changes in Thought Study), and P50NS062684 (Pacific Udall Center) and the Nancy and Buster Alvord Endowment.

\section{Authors' contributions}

BRS, KMN, CDK and KHA designed the study. BRS, KMN, LK and KLW generated the data. BRS, KMN, AP and KHA analyzed and interpreted the data. BRS and KHA wrote the manuscript. All authors read and approved the final manuscript.

\section{Funding}

These studies were funded by gifts from Karin Moe and her family, and Beverly Grossman.

\section{Availability of data and materials}

All data generated or analyzed during this study are included in this published article.

\section{Ethics approval and consent to participate}

Donated brains are from research participants in the Pacific Udall Center, the Alzheimer's Disease Research Center, and the Adult Changes in Thought Study from the University of Washington Neuropathology Core. All subjects consented to research brain autopsy as part of their specific study per protocols approved by the University of Washington Institutional Review Board.

\section{Consent for publication}

Not applicable.

\section{Competing interests}

The authors declare that they have no competing interests.

\section{Author details}

${ }^{1} \mathrm{~N}$. Bud Grossman Center for Memory Research and Care, University of Minnesota, 2101 6th Street SE, Minneapolis 55455, USA. ${ }^{2}$ Department of Neurology, University of Minnesota, Minneapolis, USA. ${ }^{3}$ Department of Medicinal Chemistry, University of Minnesota, Minneapolis, USA. ${ }^{4}$ Division of Biostatistics, School of Public Health, University of Minnesota, Minneapolis, USA. ${ }^{5}$ Department of Pathology, University of Washington, Seattle 98195, USA. ${ }^{6}$ Minneapolis VA Medical Center, Minneapolis 55417, USA.

\section{Received: 14 May 2019 Accepted: 1 July 2019}

Published online: 30 July 2019

\section{References}

1. Ahmed Z, Kalinski H, Berry M, Almasieh M, Ashush H, Slager N, Brafman A, Spivak I, Prasad N, al Ml (2011) Ocular neuroprotection by siRNA targeting caspase-2. Cell Death Dis 2:e173. https://doi.org/10.1038/cddis.2011.54

2. Carroll JB, Southwell AL, Graham RK, Lerch JP, Ehrnhoefer DE, Cao LP, Zhang WN, Deng Y, Bissada N, Henkelman RM et al (2011) Mice lacking caspase-2 are protected from behavioral changes, but not pathology, in the YAC128 model of Huntington disease. Mol Neurodegener 6:59. https://doi.org/10.11 86/1750-1326-6-59

3. Chang DW, Ditsworth D, Liu H, Srinivasula SM, Alnemri ES, Yang X (2003) Oligomerization is a general mechanism for the activation of apoptosis initiator and inflammatory procaspases. J Biol Chem 278:16466-16469. https://doi.org/10.1074/jbc.C300089200

4. Emamzadeh FN, Aojula H, McHugh PC, Allsop D (2016) Effects of different isoforms of apoE on aggregation of the alpha-synuclein protein implicated in Parkinson's disease. Neurosci Lett 618:146-151. https://doi.org/10.1016/j. neulet.2016.02.042

5. Federoff M, Jimenez-Rolando B, Nalls MA, Singleton AB (2012) A large study reveals no association between APOE and Parkinson's disease. Neurobiol Dis 46:389-392. https://doi.org/10.1016/j.nbd.2012.02.002

6. Gomperts SN, Locascio JJ, Makaretz SJ, Schultz A, Caso C, Vasdev N, Sperling R, Growdon JH, Dickerson BC, Johnson K (2016) Tau positron emission tomographic imaging in the Lewy body diseases. JAMA Neurol 73:13341341. https://doi.org/10.1001/jamaneurol.2016.3338

7. Goris A, Williams-Gray CH, Clark GR, Foltynie T, Lewis SJ, Brown J, Ban M, Spillantini MG, Compston A, Burn DJ et al (2007) Tau and alpha-synuclein in susceptibility to, and dementia in, Parkinson's disease. Ann Neurol 62:145153. https://doi.org/10.1002/ana.21192

8. Guerreiro R, Ross OA, Kun-Rodrigues C, Hernandez DG, Orme T, Eicher JD, Shepherd CE, Parkkinen L, Darwent L, Heckman MG et al (2018) Investigating the genetic architecture of dementia with Lewy bodies: a twostage genome-wide association study. Lancet Neurol 17:64-74. https://doi. org/10.1016/S1474-4422(17)30400-3

9. Hoover BR, Reed MN, Su J, Penrod RD, Kotilinek LA, Grant MK, Pitstick R, Carlson GA, Lanier LM, Yuan LL et al (2010) Tau mislocalization to dendritic spines mediates synaptic dysfunction independently of neurodegeneration. Neuron 68:1067-1081. https://doi.org/10.1016/j.neuron.2010.11.030

10. Horvath J, Herrmann FR, Burkhard PR, Bouras C, Kovari E (2013) Neuropathology of dementia in a large cohort of patients with Parkinson's disease. Parkinsonism Relat Disord 19:864-868; discussion 864. https://doi. org/10.1016/.jparkreldis.2013.05.010

11. Jean YY, Ribe EM, Pero ME, Moskalenko M, labal Z, Marks $L$, Greene LA, Troy CM (2013) Caspase-2 is essential for c-Jun transcriptional activation and Bim induction in neuron death. Biochem J 455:15-25. https://doi.org/10.1 042/BJ20130556

12. Labbe C, Heckman MG, Lorenzo-Betancor O, Soto-Ortolaza Al, Walton RL, Murray ME, Allen M, Uitti RJ, Wszolek ZK, Smith GE et al (2016) MAPT haplotype $\mathrm{H} 1 \mathrm{G}$ is associated with increased risk of dementia with Lewy bodies. Alzheimers Dement 12:1297-1304. https:/doi.org/10.1016/j.jalz.2016.05.002

13. Lopez-Cruzan M, Sharma R, Tiwari M, Karbach S, Holstein D, Martin CR, Lechleiter JD, Herman B (2016) Caspase-2 resides in the mitochondria and mediates apoptosis directly from the mitochondrial compartment. Cell Death Discov 2. https://doi.org/10.1038/cddiscovery.2016.5

14. Parsian A, Racette B, Goldsmith LJ, Perlmutter JS (2002) Parkinson's disease and apolipoprotein E: possible association with dementia but not age at onset. Genomics 79:458-461. https://doi.org/10.1006/geno.2002.6707

15. Pozueta J, Lefort R, Ribe EM, Troy CM, Arancio O, Shelanski M (2013) Caspase-2 is required for dendritic spine and behavioural alterations in J20 APP transgenic mice. Nat Commun 4:1939. https://doi.org/10.1038/ncomms2927

16. Seto-Salvia N, Clarimon J, Pagonabarraga J, Pascual-Sedano B, Campolongo A, Combarros O, Mateo Jl, Regana D, Martinez-Corral M, al MM (2011) Dementia risk in Parkinson disease: disentangling the role of MAPT haplotypes. Arch Neurol 68:359-364. https://doi.org/10.1001/archneurol.2011.17

17. Shi Y, Yamada K, Liddelow SA, Smith ST, Zhao L, Luo W, Tsai RM, Spina S, Grinberg LT, Rojas JC et al (2017) ApoE4 markedly exacerbates tau-mediated neurodegeneration in a mouse model of tauopathy. Nature 549:523-527. https://doi.org/10.1038/nature24016

18. Shimohama S, Tanino H, Fujimoto S (1999) Changes in caspase expression in Alzheimer's disease: comparison with development and aging. Biochem Biophys Res Commun 256:381-384. https://doi.org/10.1006/bbrc.1999.0344

19. Tiwari M, Herman B, Morgan WW (2011) A knockout of the caspase 2 gene produces increased resistance of the nigrostriatal dopaminergic pathway to MPTP-induced toxicity. Exp Neurol 229:421-428. https://doi.org/10.1016/j. expneurol.2011.03.009

20. Troy CM, Rabacchi SA, Friedman WJ, Frappier TF, Brown K, Shelanski ML (2000) Caspase-2 mediates neuronal cell death induced by beta-amyloid. J Neurosci 20:1386-1392

21. Tsuang D, Leverenz JB, Lopez OL, Hamilton RL, Bennett DA, Schneider JA, Buchman AS, Larson EB, Crane PK, Kaye JA et al (2013) APOE epsilon4 increases risk for dementia in pure synucleinopathies. JAMA Neurol 70:223228. https://doi.org/10.1001/jamaneurol.2013.600

22. Zhang Y, Padalecki SS, Chaudhuri AR, De Waal E, Goins BA, Grubbs B, lkeno $Y$, Richardson A, Mundy GR, Herman B (2007) Caspase-2 deficiency enhances aging-related traits in mice. Mech Ageing Dev 128:213-221. https://doi.org/10.1016/j.mad.2006.11.030

23. Zhao X, Kotilinek LA, Smith B, Hlynialuk C, Zahs K, Ramsden M, Cleary J, Ashe KH (2016) Caspase-2 cleavage of tau reversibly impairs memory. Nat Med 22:1268-1276. https://doi.org/10.1038/nm.4199

\section{Publisher's Note}

Springer Nature remains neutral with regard to jurisdictional claims in published maps and institutional affiliations. 